\title{
Oral Health and Cognitive Function in Older Adults: A Systematic Review
}

\author{
Matthew R. Nangle ${ }^{a} \quad$ Julia Riches ${ }^{b}$ Sarah A. Grainger ${ }^{b}$ Nithin Manchery ${ }^{a}$ \\ Perminder S. Sachdev ${ }^{c, d}$ Julie D. Henry ${ }^{b}$

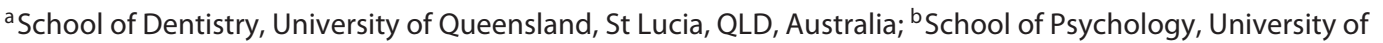 \\ Queensland, St Lucia, QLD, Australia; 'C Centre for Healthy Brain Ageing, University of New South Wales, Sydney, \\ NSW, Australia; ${ }^{d}$ Neuropsychiatric Institute, Prince of Wales Hospital, Randwick, NSW, Australia
}

\section{Keywords}

Systematic review · Older adults · Oral health · DSM-5 ·

Cognitive function

\begin{abstract}
Background: It has often been argued that there is a relationship between oral health and cognitive decline in late adulthood, but a recent systematic review concluded that it was unclear "how or whether" any relationship exists. However, most of the studies that contributed to this review operationalised cognitive function using a brief cognitive screen and/or dementia status. Objective: An updated systematic review was conducted that focused on how oral health relates to specific cognitive abilities in older adults (specifically, the neurocognitive domains specified in the DSM-5: learning and memory, perceptual motor function, language, executive function, complex attention, and social cognition). Methods: A systematic review was undertaken and completed in August 2018. From a total of 1,304 potentially relevant articles, 23 were identified that assessed oral health and at least one of the specific cognitive domains in an older adult cohort. Results: The most consistent relationships were identified with learning and memory, complex attention, and executive function. For each of these cogni-
\end{abstract}

tive domains, most studies identified significant unadjusted associations with oral health; where adjustments for covariates were made, at least one of the associations with oral health remained significant in half or more of the studies. Results were less clear for the domains of language and perceptual motor function. No study assessed the relationship between social cognition and oral health. Conclusions: This systematic review provides evidence of an association between learning and memory, complex attention, and executive function with oral health in old age. Gaining a detailed picture of how specific types of cognitive decline relate to oral health has potential implications for earlier identification of older adults who experience oral health problems, and may also inform the development of more effective interventions focused on enhancing oral health outcomes in this group.

๑ 2019 S. Karger AG, Basel

\section{Introduction}

How cognitive function relates to oral health in late adulthood has been the focus of considerable empirical research in recent years [1]. It has been frequently argued that the relationship between these two constructs is both

\section{KARGER}

(c) 2019 S. Karger AG, Basel

E-Mail karger@karger.com

www.karger.com/ger
Julie D. Henry

School of Psychology

University of Queensland

St Lucia, QLD 4072 (Australia)

E-Mail julie.henry@uq.edu.au 


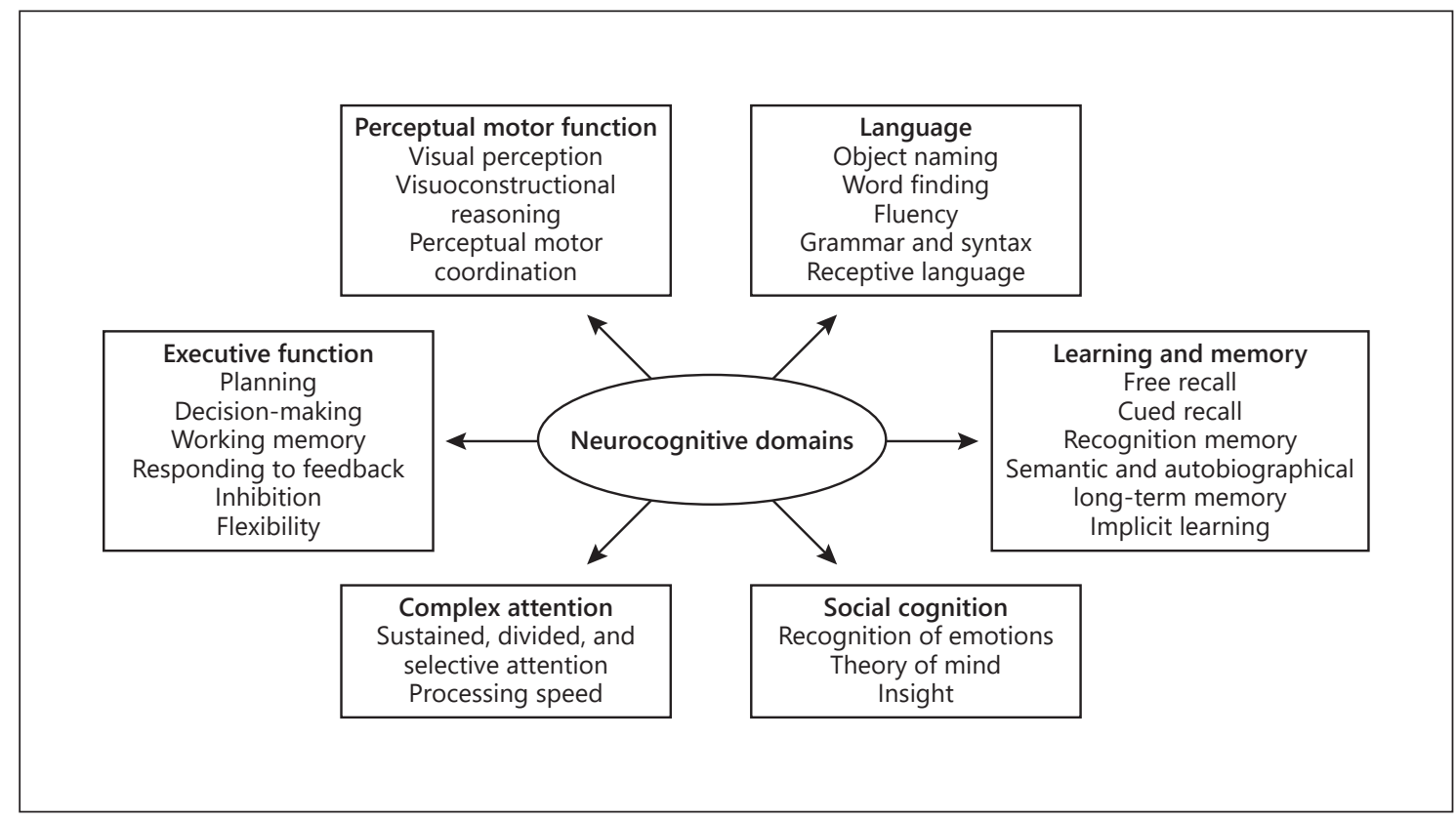

Fig. 1. Neurocognitive domains. The DSM-5 defines 6 key domains of cognitive function, and each of these has subdomains. Figure 1 is also published as part of the review of Sachdev et al. [12] focused on the classification of neurocognitive disorders in the DSM-5 and has been reproduced here with permission from Nature Reviews Neurology.

clear and consistent, with poorer oral health reliably linked to greater cognitive decline. For instance, Peres et al. [2] noted that "regardless of methodological designs and strategies... a consistent and low-to-moderate association between these events has been demonstrated" ( $p$. 877). Indeed, there is both cross-sectional and longitudinal research evidence which shows that older adults present with lower cognitive performance when there are specific oral health conditions, such as periodontal disease $[3,4]$, dental caries [5], difficulties with masticatory function $[6,7]$, and tooth loss [8].

In terms of explaining these relationships, most studies to date have used cross-sectional research methods which limit inferences about causality. However, it has been suggested that poor oral health might lead to cognitive decline via specific biological mechanisms such as common inflammatory pathways or reduced nutritional intake [9]. However, the opposite direction of causality is equally plausible, as a straightforward argument can also be presented for how cognitive decline might negatively impact oral health via behavioural changes such as reduced attention to oral hygiene or inadequate use of dental health services. Consistent with this latter possibility, Brennan and Strauss [10] note that cognitive impairment in older adulthood is often followed by rapid oral health deterioration, as functionality in daily life decreases. There is also the potential for very specific cognitive impairments such as apraxia (which disrupts motor planning) to decrease an individual's ability to effectively engage in oral hygiene [4].

Evidence suggesting that there may be a potential relationship between oral health and cognitive function has proven so influential that it has begun to be included in health care guidelines. For instance, Chen et al.[11] has argued that, "given the relationship between cognitive impairment and oral health, it is essential that dentally related function assessment be a standard part of geriatric dental care" (p. 580). In light of this argument that a relationship exists, the findings of a recent systematic review of 56 studies ( 40 cross sectional, 16 longitudinal) were unexpected. Specifically, Wu et al. [1] reported that while some studies found evidence of an association between oral health and cognitive function, others did not, and concluded that, "it is unclear how or whether oral health conditions and cognitive status are related" (p. 739, italics added).

However, an important consideration is that many of the studies that contributed to the review of $\mathrm{Wu}$ et al. [1] 
Fig. 2. Adapted PRISMA diagram illustrat-

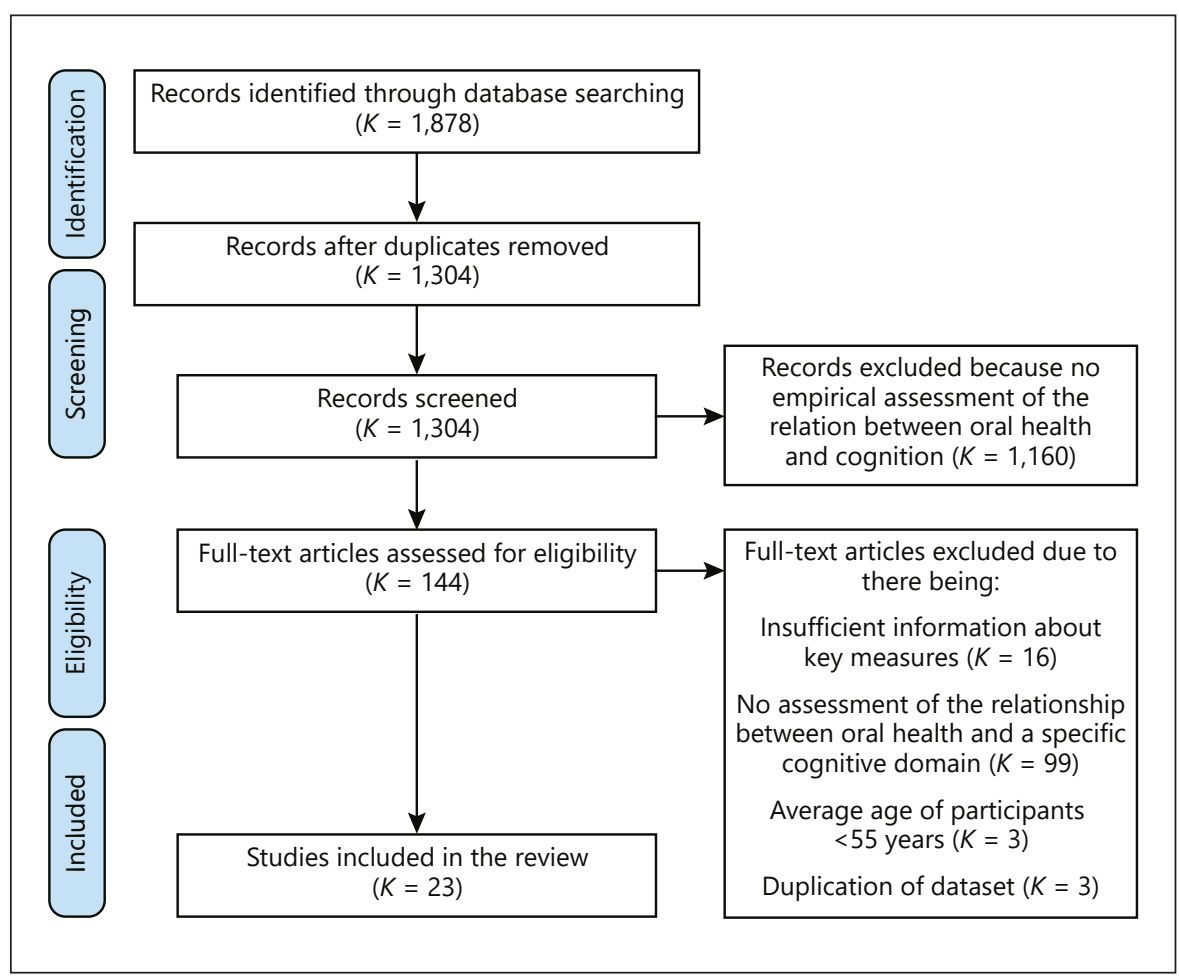
ing the study selection process.

operationalised cognitive function using a brief cognitive screen and/or the presence of dementia (via self-report, diagnostic criteria, and/or from medical records). While these types of data provide an indication of whether older adults exhibit potential cognitive impairment, they provide little insight into the nature of any cognitive difficulties. Another important consideration is that, as noted, to date most studies focused on the relationship between cognitive function and oral health have used cross-sectional research methods, which do not allow inferences to be made about the temporality of any association and, consequently, causality.

The DSM-5 identifies 6 key domains of neurocognitive function, and each of these includes multiple subdomains shown in Figure 1 (reproduced from Sachdev et al. [12]). Since specific types of cognitive abilities may differ in their relationship to oral health, the goal of this systematic review was to establish more precisely what is known about specific cognitive functions and oral health by using the DSM-5 classification system to provide a structure. Given that only longitudinal research evidence speaks to potential causality, in structuring this review, a distinction will also be made between cross-sectional and longitudinal research evidence.

Cognitive Function and Oral Health

\section{Methods}

The methodological approach was based on the PRISMA guidelines. The following databases were searched to identify studies that assessed oral health and cognitive function, and reported data for older adults: PubMed, Complete Scopus, PsychINFO, and Web of Science. The search was completed in August 2018. The databases were searched for English language journal articles using the following terms and/or keywords: (oral health OR tooth loss OR dental caries OR periodontal disease OR oral hygiene) AND (memory OR executive OR language OR aphasia OR apraxia OR attention OR cognition OR reasoning). The PubMed database identified 681 articles (filter: aged 55+), Scopus identified 461 articles (filter: aged), Web of Science identified 667 articles (filter: adult* or elder*), and PsychInfo identified 69 articles (filter: aged $55+$ ). Duplicate articles were identified using a combination of referencing software and hand searching. After removing 574 duplicates, 1,304 unique articles remained. A further 1,160 articles were then removed based on title/abstract review, because they did not include any empirical assessment of the relationship between oral health and cognitive function.

The remaining 144 articles were subjected to full-text screening, with the following exclusionary criteria applied: (1) the article did not contain adequate information on cognitive or oral health measures, (2) the article did not examine the relationship between a specific cognitive function and oral health status (i.e., the authors used a broad cognitive screen and not a specific cognitive measure), and (3) the average age of participants was less than 55 years. As shown in Figure 2, 16 articles were excluded due to criterion I. The second and third criteria resulted in the exclusion of 99 and 3 


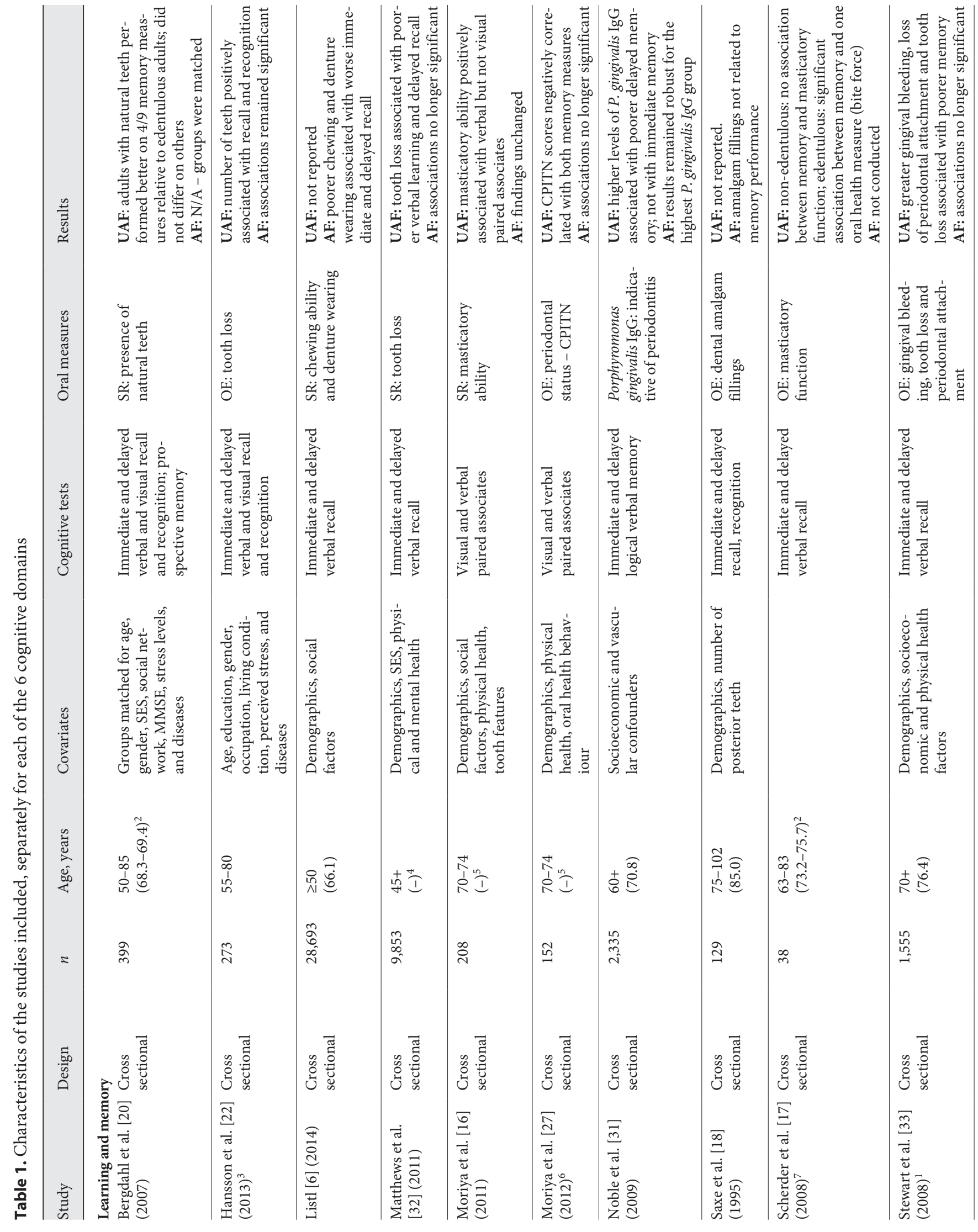




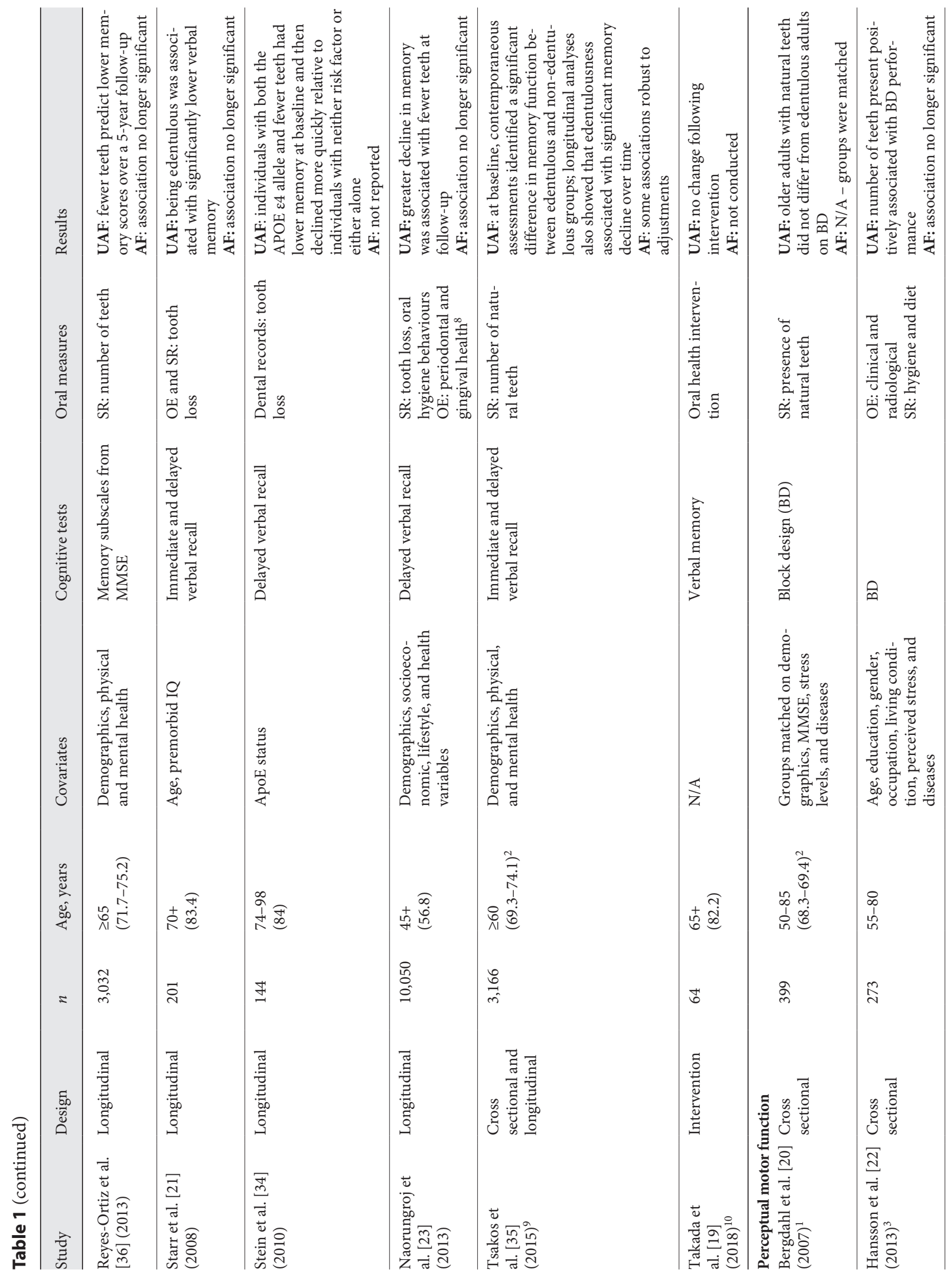




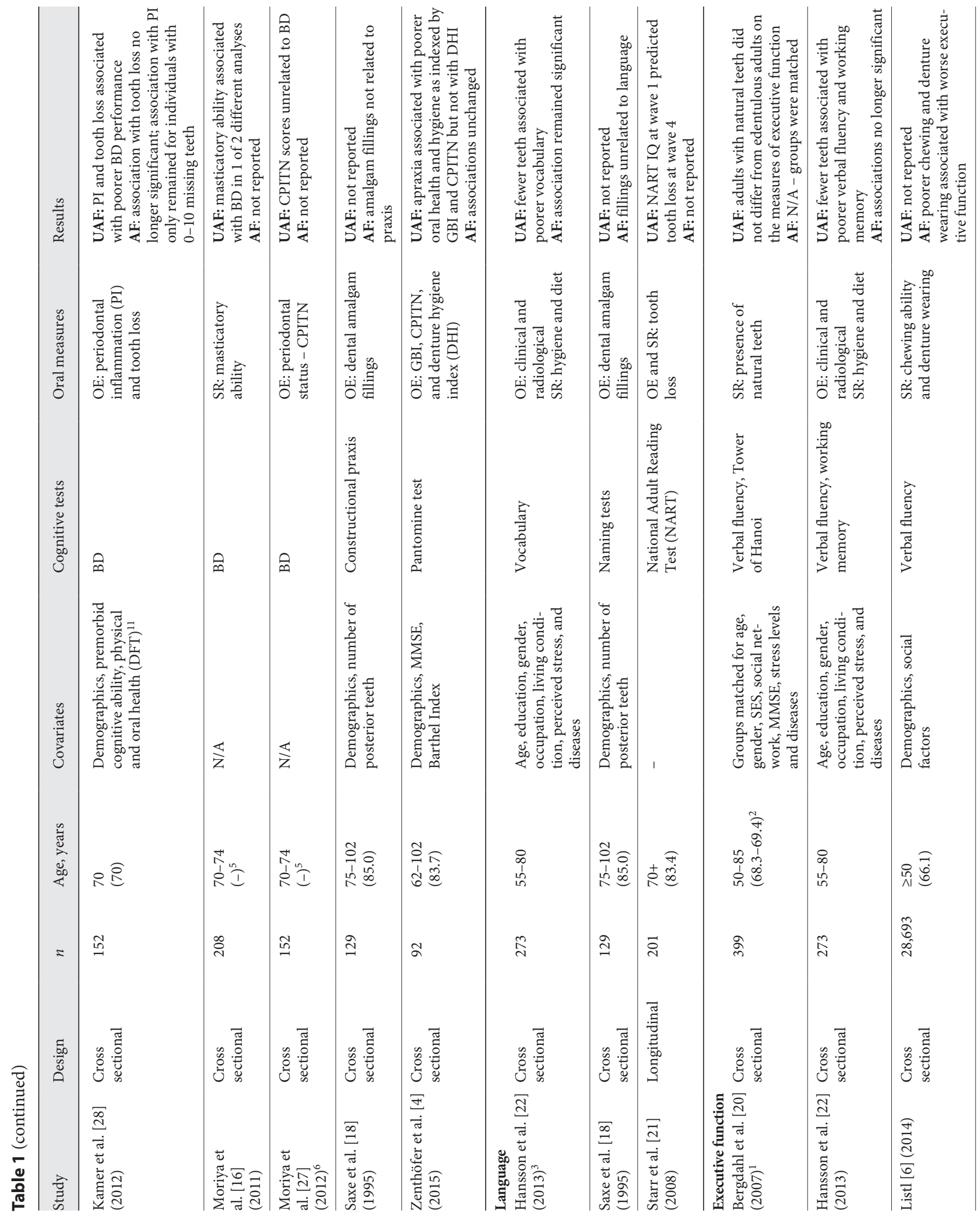




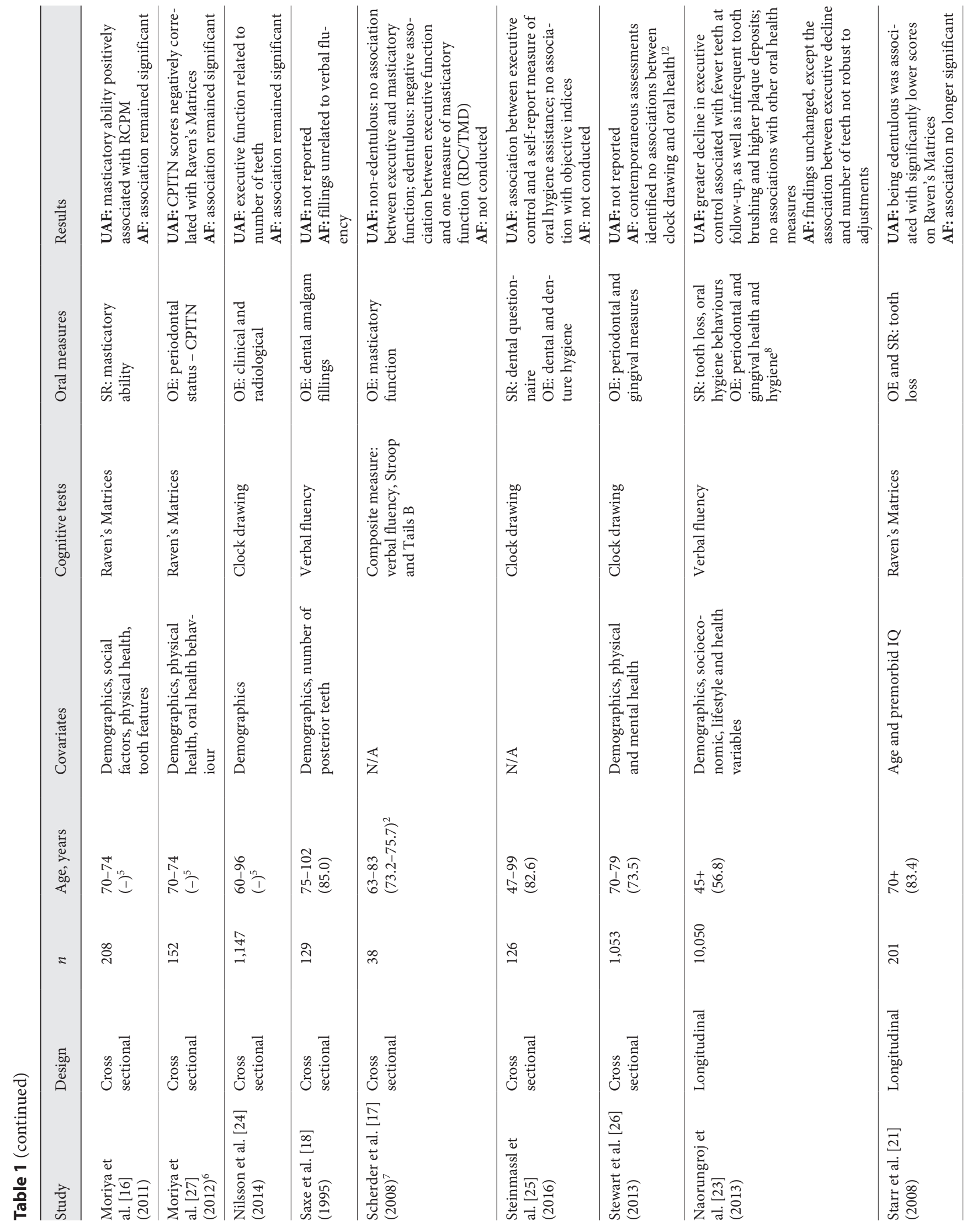




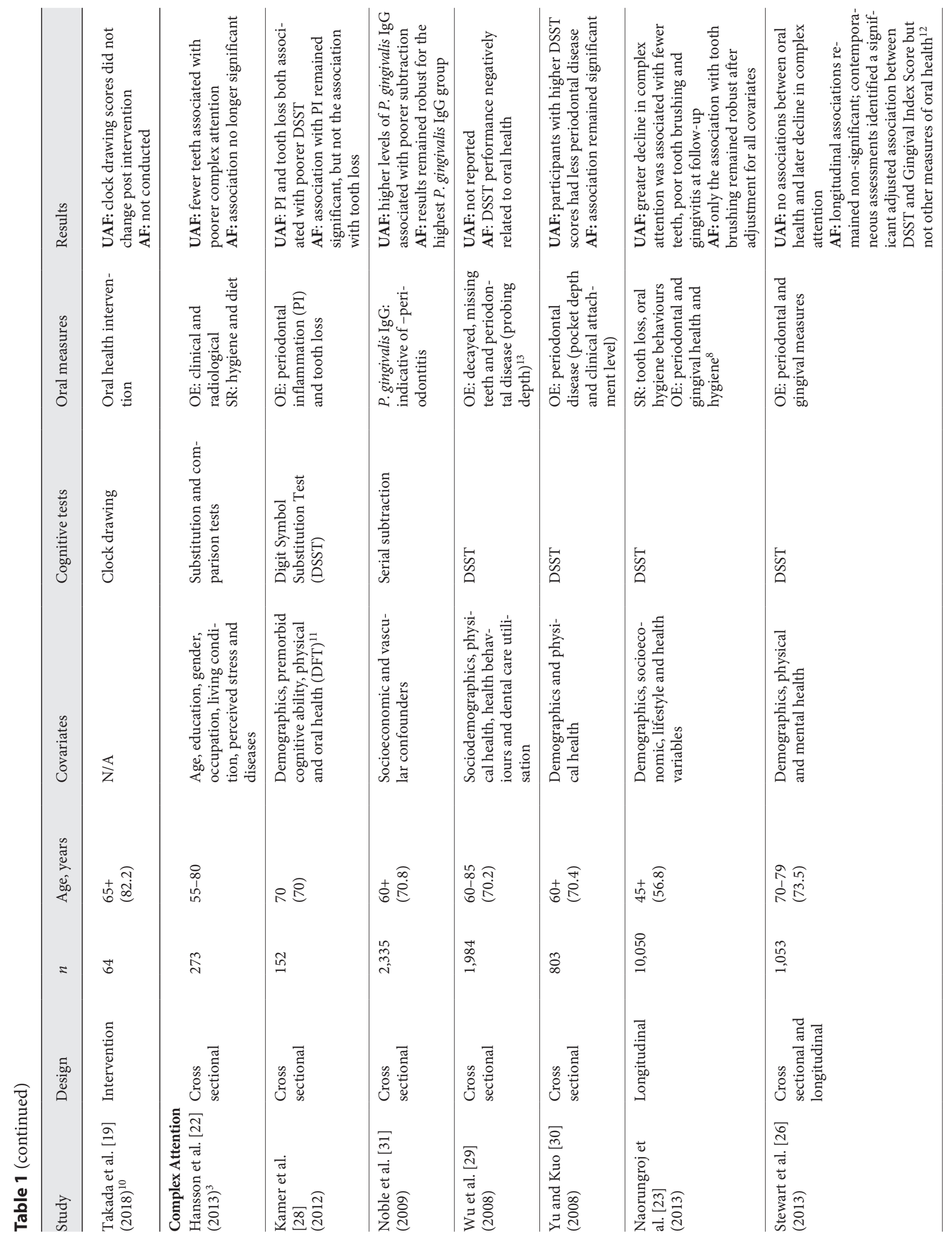




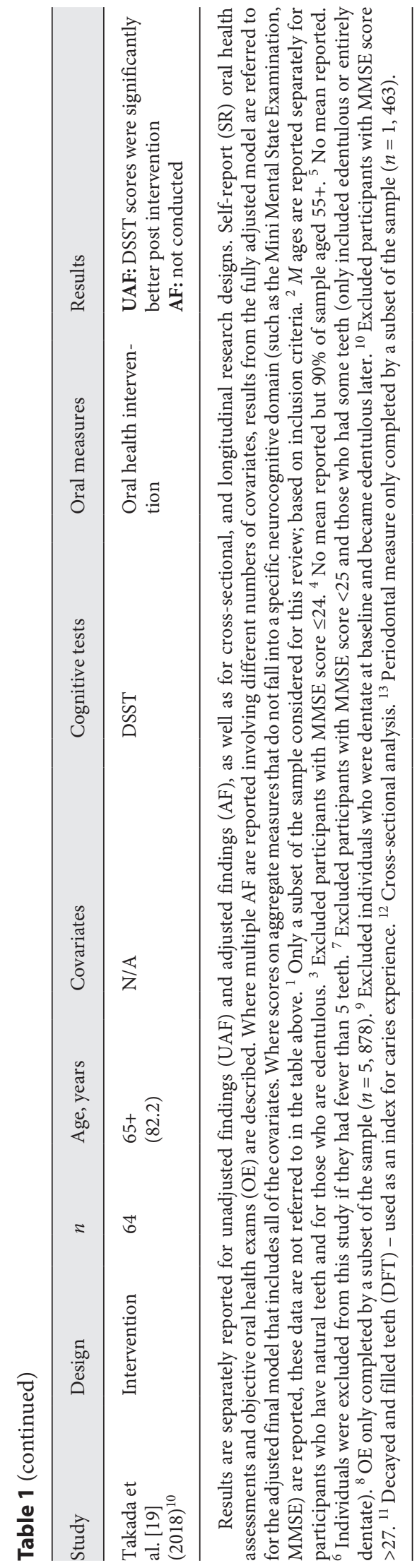

Cognitive Function and Oral Health articles, respectively. Three additional articles were excluded from the analysis, as they used an identical data set and measures to another included article [13-15]. This left a total of 23 articles.

With respect to data extraction, one author screened the abstracts of all articles to exclude obviously irrelevant articles. Two authors independently screened the remaining articles and extracted data. A third reviewer critically appraised and verified the data. The key information extracted was in relation to authors, date of publication, study or data collection date, study location, sample demographics, cognitive measures, oral health measures, covariates, analytical procedures, and key findings.

\section{Results of Literature Search}

A summary of each included article can be found in Table 1. The earliest study was published in 1995, the most recent in 2018. Predominantly developed countries are represented (USA, $K=10$; Sweden, $K=3$; Japan, $K=3$; Germany, $K=1$, UK, $K=2$; the Netherlands, $K=1$; Denmark, $K=1$; Austria, $K=1$; and a European consortium, $K=1$ ). Nineteen of the studies were conducted with community-based individuals, and 2 focused on nursing or care home populations. The final 2 articles used data from the Nun Study of Ageing and Alzheimer's disease.

Sample size ranged from small $(n=38)$ to extremely large $(n=$ $28,693)$. Sixteen articles were cross-sectional studies, 6 longitudinal, and 1 was an oral health intervention. Five studies collected primary data, 1 analysed health records, and 17 performed secondary analyses on existing data sets. Of these, 4 used data from the National Health and Nutrition Examination Survey, 2 from the Betula Prospective Cohort Study, and 2 from the Nun Study. However, none of these papers presented identical data from the same cohort.

\section{Oral Health Measures}

Researchers used a variety of sources to index oral health, including dental records, dental examinations, and self-report measures. Thirteen articles used objective oral exams or measures, 6 used self-report measures only, and 4 used both objective and selfreport measures. Tooth loss was the most common measure of oral health, appearing in 12 of the 23 articles. This was followed by measures of periodontal health and hygiene, which were used in 11 articles. These included standardized oral health indices such as the Community Periodontal Index of Treatment Needs (CPITN) and the Gingival Bleeding Index (GBI). Functional dental status, such as masticatory ability or denture wearing, was also used to assess oral health in 3 articles [6, 16, 17]. Saxe et al. [18] specifically looked at the amount of dental amalgam in relation to cognitive function, and Takada et al. [19] assessed cognitive ability before and after an oral health intervention.

\section{Cognitive Measures}

As noted, the classification system provided by the DSM-5, which identifies 6 key domains of neurocognitive function, was used. As shown in Figure 1, each of these domains also has subdomains, and consequently for most cognitive measures it was straightforward to allocate a specific test to a particular subdomain. On the rare occasions where a non-standardized task was used or composite measures created, this is noted.

Learning and Memory. The relation between oral health and various types of memory were examined in 16 studies $(10$ cross sectional, 4 longitudinal, 1 both cross-sectional and longitudinal, 
and 1 intervention). All 16 studies used verbal memory tasks, with a minority $(K=4)$ also investigating visual memory. In both verbal and visual tests, authors included a combination of immediate, delayed, and recognition memory. Only 1 study examined prospective memory [20].

Perceptual Motor Function. Perceptual motor function was assessed in 7 studies, all cross-sectional studies. Most studies (5) tested perceptual motor function using block design. One study used a constructional praxis task to measure constructional apraxia [18], and another used the pantomime test to assess ideomotor apraxia [4].

Language. Language ability was assessed in 3 studies, 2 cross sectional and 1 longitudinal. One study used the Boston Naming Test and Object Naming test [18], another used the National Adult Reading Test [21], and a 30-item multiple-choice vocabulary test was used in the final study [22].

Executive Function. Executive functioning was assessed in 13 articles, 10 cross sectional, 2 longitudinal, and 1 intervention. Five studies used verbal fluency tasks $[6,18,20,22,23]$, and 4 used the clock drawing test $[19,24-26]$. One article used a composite measure of executive function made up of the Trail Making Test, the Stroop and verbal fluency test [17]. Raven's matrices were used in 3 studies [16, 21,27]. One study used the Tower of Hanoi task [20].

Complex Attention. Eight studies assessed complex attention, 5 cross sectional, 2 longitudinal, and 1 intervention. The Symbol Digit Modalities Test was used to index complex attention in 6 studies [19, 23, 26, 28-30]. One study used a serial substraction test [31], and another used both substitution and comparison tests [22].

Social Cognition. None of the 23 studies assessed the relationship between oral health and social cognition.

\section{Covariates}

A wide range of covariates were used. These included demographic characteristics, socioeconomic status, specific health conditions and risk factors, health behaviours (e.g., alcohol consumption or physical activity), mental health, physical health, place of residence, dental care utilisation, social functioning, genetic risk factors, and medical or dental insurance. None of the studies included all of these covariates, and there was rarely any uniformity of measurement.

\section{Relationship between Oral Health and Specific Cognitive} Functions

Learning and Memory. For the 10 cross-sectional studies, where unadjusted findings were reported (8 of the studies), all studies identified a significant association between learning and memory with at least 1 oral health measure and/or for at least some of the participant subgroups. Where adjusted findings were also reported (for 6 of these 8 studies) covarying for a range of different variables, at least some of the reported associations remained for 3 of the studies $[16,22,31]$, whereas for the other 3 studies, the associations were no longer significant $[27,32,33]$. For the 2 studies that only reported adjusted relationships, a significant association was identified for 1 of these studies [6] but not the other [18]. Taken together, there is good evidence for an association between learning and memory with oral health when only non-adjusted findings are assessed, and at least some associations remained in approximately half of the studies that adjusted for secondary variables.
Four of the 5 longitudinal studies examined whether oral health predicted subsequent memory decline [21,34-36]. For all 4, a significant relationship was identified between oral health and subsequent memory decline. In 1, it was the combination of poor oral health in addition to the ApoE 4 allele which most strongly predicted subsequent decline [34]. For the remaining studies, where adjustments for covariates were applied, the association between oral health and later memory decline was no longer significant in 2 studies [21,36], but remained significant in the third [35]. In the only study to examine the reverse direction of causality, greater decline in memory did predict poorer oral health, but this relationship was no longer significant after adjustment for covariates [23].

Perceptual Motor Function. All 7 studies to investigate the relationship between oral health and perceptual motor function used cross-sectional research methods. Where unadjusted findings were reported (for 6 of the 7 studies), at least 1 significant association was identified in 4 of the studies $[4,16,22,28]$. Where adjusted findings were then reported (for 3 of these 4 studies), this relationship was eliminated in 1 [22], and unchanged for a second [4]. For the third study, an association persisted with 1 oral health measure, but only for 1 subgroup of older adults [28]. For the 1 study that only reported adjusted (and not unadjusted) findings, no relationship was identified between perceptual motor function and oral health [18]. Taken together, the results with regard to perceptual motor function and oral health are mixed.

Language. Only 2 cross-sectional studies investigated the relationship between oral health and language. In 1, a significant association with oral health was identified which remained robust to covariates [22]; however, the second study reported adjusted findings only, and identified no association [18]. The only study to use longitudinal research methods showed that performance on a measure of language ability at Wave 1 was a significant predictor of tooth loss at Wave 4 [21]. Two of the 3 studies, therefore, suggest that there may be an association between language and oral health, but conclusions here are cautious given the small number of studies.

Executive Function. For the 10 cross-sectional studies, where unadjusted findings were reported (for 7 of the studies), a significant association between executive function and oral health was identified in 6 of these studies [16, 17, 22, 24, 25, 27]. For 4 of these 6 studies, adjusted findings were also reported. At least some associations between oral health and executive control remained significant in 3 of these 4 studies [16, 24,27], but this was not the case for the fourth [22]. Of the 3 studies that reported only adjusted findings, a significant association between oral health and executive function was identified in 1 study [6] but not in the other 2 [18, 26]. Taken together, most studies indicate that there is an association between executive control and oral health when unadjusted findings are assessed, and this relationship persists in approximately half of the studies that adjust for covariates.

Only 2 studies used longitudinal research methods to investigate the relationship between executive control and oral health. Naorungroj et al. [23] showed that greater decline in executive control was significantly related to some but not all indices of oral health at follow-up in both unadjusted and adjusted models. Starr et al. [21] found that although tooth loss was associated with subsequent executive decline, this association was no longer significant after taking original intelligence level into account.

Complex Attention. Of the 6 cross-sectional studies that assessed complex attention, 4 reported unadjusted findings, and a 


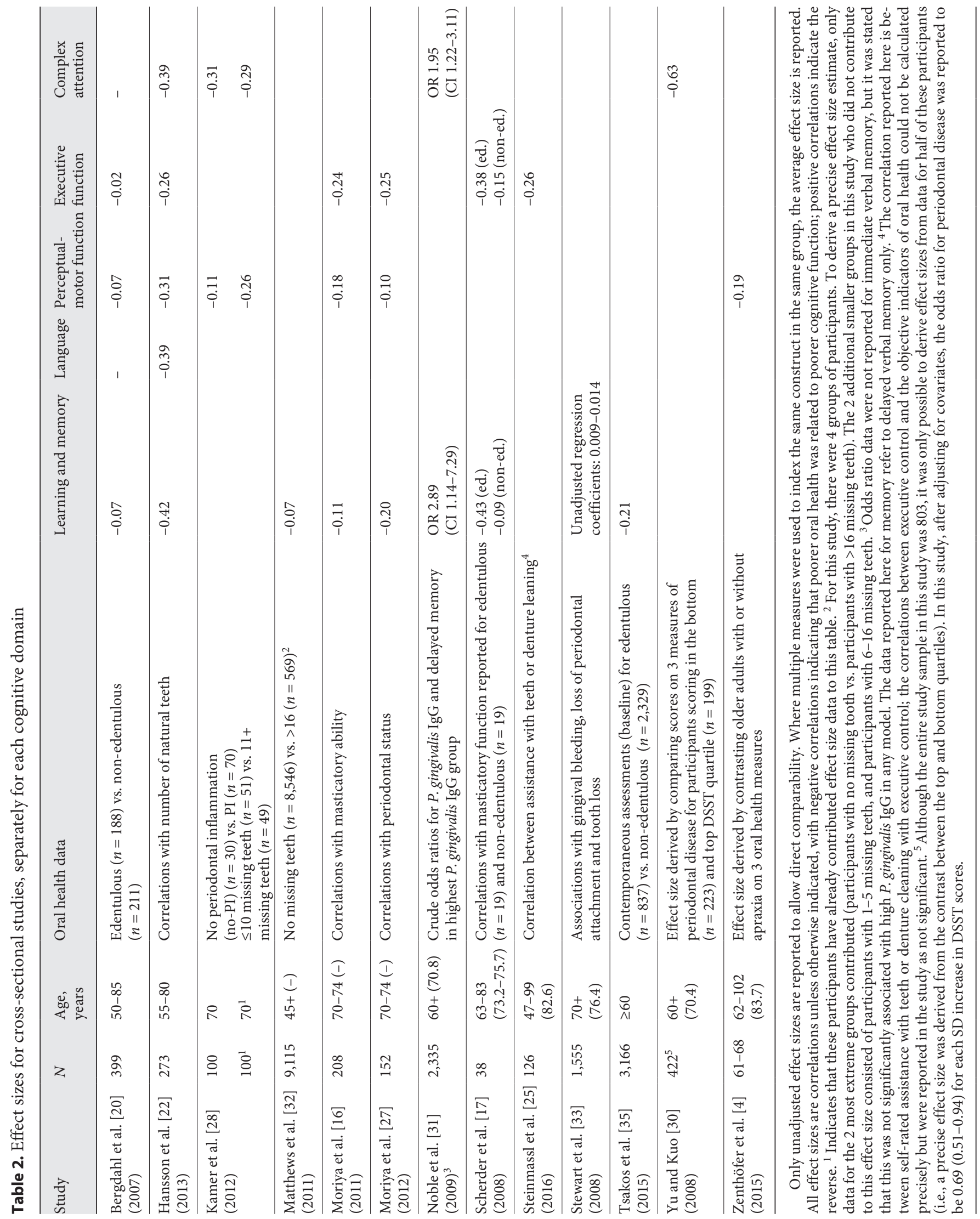


significant association with oral health was identified in all 4 of these studies [22, 28, 30, 31]. These 4 studies also reported adjusted findings; for 1 of these studies, no associations were found following adjustment [22], while for the other 3 studies, at least some associations remained significant $[28,30,31]$. For the 2 cross-sectional studies that only reported adjusted findings, complex attention was negatively related to oral health $[26,29]$. Taken together, there is good cross-sectional research evidence that there is an association between oral health and complex attention, and this relationship is robust to adjustments for covariates.

Two longitudinal studies assessed the relationship with oral health. One of these showed that greater decline in complex attention was associated with indices of oral health, and that one of these associations remained significant following adjustments [23]. The other study found that oral health measures at year 2 did not predict subsequent decline in complex attention [adjusted or unadjusted; 26].

\section{Magnitude of Effect Sizes}

For the cross-sectional studies that reported effect sizes, or data that allowed precise effect sizes to be derived, Table 2 reports effect sizes for each study, separately for each cognitive domain. Where more than a single measure was used to index a cognitive domain, the average effect size was derived. It can be seen that where effect size data were reported or could be precisely calculated, the magnitude of effects was typically small or moderate in magnitude.

\section{Discussion}

This systematic review of 23 studies provides the first integration of more than 2 decades of empirical research focused on identifying how specific cognitive abilities relate to oral health in old age. As noted earlier, assessment of individual cognitive domains has direct clinical importance because if specific associations do exist, this information might help to identify which particular older adults are likely to also present with oral health problems, and may also potentially inform how oral health programmes should be presented to optimise oral health outcomes in this group. This latter consideration is particularly important since as concluded in a recent systematic review, "There is a clear paucity of well-designed research studies to investigate strategies to improve the oral health of elderly people with cognitive impairment" [37].

The results indicate that learning and memory was the most commonly assessed cognitive domain, and when only unadjusted findings were considered, all 8 of the cross-sectional studies and all 5 of the longitudinal studies identified a significant association. These data, therefore, suggest that difficulties with learning and memory are related to poorer oral health. Moreover, because at least one or more of the associations remained in approximately half of the studies that adjusted for covariates, it appears that this relationship can be, but is not always, accounted for by secondary variables. Importantly, although subjective memory complaints are very common in late adulthood, all of the studies that contributed to this review used objective indicators. These data, therefore, do not speak to whether subjective memory complaints are linked to poorer oral health, but they do indicate that in circumstances where an individual exhibits low scores on measures of learning and memory, an oral health assessment should be recommended.

Evidence for an association with oral health was also evident for the domains of complex attention and executive control. For complex attention, almost all studies that reported unadjusted findings and all but 1 of the studies that reported adjusted findings identified at least some association with oral health. Nearly all of the studies contributing to this review used the same measure of complex attention, the Digit Symbol Substitution Test (DSST). For executive control, findings were more mixed (and this may be partially attributable to there being greater heterogeneity in the measures used to operationalize this construct). Nevertheless, again, most studies that reported unadjusted findings identified at least some association, and some relationship remained significant in approximately half of the studies following adjustment for covariates.

Both complex attention and executive control have previously been identified as predictors of functional outcomes. This is unsurprising given that complex attention encompasses a number of cognitive operations that are critical in a range of everyday contexts, such as sustained attention, divided attention, selective attention, and processing speed. Analogously, executive control is likely to be critical in many everyday contexts, encompassing higher-order control operations such as inhibition, planning, mental set-shifting and self-initiated processing. Indeed, for inpatient stroke rehabilitation, complex attention has been identified as one of the most important predictors of rehabilitation gain and instrumental activities of daily living (IADL) functioning at discharge [38], while executive dysfunction has been shown to predict IADLs in older adults with Alzheimer's disease, even after controlling for demographic variables and depression [39].

Therefore, one interesting possibility is that difficulties with complex attention and/or executive control might contribute to poor oral health via what has been termed reduced dental-related functionality [11]. Indeed, in the only longitudinal study to date to test whether these relationships exist, poorer complex attention and executive control independently predicted poorer oral health at fol- 
low-up, and this effect was robust across both adjusted and unadjusted models [23]. By contrast, in the only 2 studies to investigate the reverse direction of causality, oral health did not predict subsequent either adjusted or unadjusted decline in complex attention [26], or executive decline after taking original intelligence into account [21]. Clearly however, conclusions based on such a small number of longitudinal studies need to be cautious, and further longitudinal studies are needed to directly test these competing possibilities.

For the other 3 cognitive domains, it was more difficult to reach a clear conclusion about whether any potential relationship with oral health exists at all. This is because there were either few studies (language), no studies (social cognition), or because findings were mixed (perceptual motor function). Further work is therefore needed to explore how oral health relates to these 3 specific cognitive domains. Indeed, this review in particular highlights the need for future work in this field to consider social cognition, a neurocognitive domain that is subject to decline in both normal and abnormal adult ageing [40,41].

Finally, limitations of this review are acknowledged. First, although we performed a comprehensive literature search, unpublished studies did not contribute. Second, although for all studies objective, standardized indicators of cognitive function were used, studies varied considerably in how they operationalized oral health, ranging from comprehensive, objective oral exams, to single-item self-report measures of tooth loss. Third, studies that reported adjusted findings varied in both the number and nature of their covariates. Fourth, each cognitive test was allocated to only 1 specific cognitive domain. This approach was used because it is important to be consistent and precise with how cognitive tests are interpreted if we are to gain a clearer understanding of how oral health relates to specific cognitive domains. However, all cognitive assessments are to at least some extent multifactorial and also impose demands on general ability, and this needs to be acknowledged. By ignoring the common variance that cognitive tests share, there is always the danger that this approach over-emphasises specificity that does not exist. Finally, while the current review identified an association between oral health and specific cognitive domains, it can provide only limited insights with respect to causality because the majority of studies that contributed to this review were cross sectional.

\section{Conclusion}

This review provides evidence that the specific cognitive domains of learning and memory, complex attention, and executive control are related to oral health in late adulthood. Further work is now needed using prospective longitudinal designs that incorporate assessments of specific cognitive functions to better understand issues of potential causality and directions of effects.

\section{Acknowledgement}

This work was funded by an Australian Dental Research Foundation Grant awarded to Grainger, Nangle, and Henry. Henry was also supported by an Australian Research Council Future Fellowship.

\section{Statement of Ethics}

This work was conducted in accordance with accepted standards of ethics.

\section{Disclosure Statement}

None of the authors has any conflicts of interest to disclose.

\section{References}

1 Wu B, Fillenbaum GG, Plassman BL, Guo L. Association Between Oral Health and Cognitive Status: A Systematic Review. J Am Geriatr Soc. 2016 Aug;64(8):1752-1752.

2 Peres MA, Bastos JL, Watt RG, Xavier AJ, Barbato PR, D'Orsi E. Tooth loss is associated with severe cognitive impairment among older people: findings from a population-based study in Brazil. Aging Ment Health. 2015; 19(10):876-84
3 Martande SS, Pradeep AR, Singh SP, Kumari M, Suke DK, Raju AP, et al. Periodontal health condition in patients with Alzheimer's disease. Am J Alzheimers Dis Other Demen. 2014 Sep;29(6):498-502.

4 Zenthöfer A, Navratil SD, Rammelsberg P, Cabrera T, Gorenc L, Urbanowitsch N, et al. Oral health and apraxia among institutionalized elderly people-a pilot study. Acta Odontol Scand. 2015 Feb;73(2):150-5.
5 Chen X, Clark JJ, Chen H, Naorungroj S. Cognitive impairment, oral self-care function and dental caries severity in community-dwelling older adults. Gerodontology. 2015 Mar;32(1): 53-61.

6 Listl S. Oral health conditions and cognitive functioning in middle and later adulthood. BMC Oral Health. 2014 Jun;14(1):70. 
7 Weijenberg RA, Scherder EJ, Lobbezoo F. Mastication for the mind-the relationship between mastication and cognition in ageing and dementia. Neurosci Biobehav Rev. 2011 Jan;35(3):483-97.

8 Luo J, Wu B, Zhao Q, Guo Q, Meng H, Yu L, et al. Association between tooth loss and cognitive function among 3063 Chinese older adults: a community-based study. PLoS One. 2015 Mar; 10(3):e0120986.

9 Noble JM, Scarmeas N, Papapanou PN: Poor oral health as a chronic, potentially modifiable dementia risk factor: Review of the literature. Curr Neurol Neurosci Rep. 2013 Oct; 13(10):384.

10 Brennan LJ, Strauss J. Cognitive impairment in older adults and oral health considerations: treatment and management. Dent Clin North Am. 2014 Oct;58(4):815-28.

11 Chen X, Zimmerman S, Potter GG, Sloane PD, Cohen LW, Reed D. Assessment of dentally related function in individuals with cognitive impairment: The Dental Activities Test. J Am Geriatr Soc. 2017 Mar;65(3):580-5.

12 Sachdev PS, Blacker D, Blazer DG, Ganguli $\mathrm{M}$, Jeste DV, Paulsen JS, et al. Classifying neurocognitive disorders: the DSM-5 approach. Nat Rev Neurol. 2014 Nov;10(11):634-42.

13 Naorungroj S, Schoenbach VJ, Wruck L, Mosley TH, Gottesman RF, Alonso A, et al. Tooth loss, periodontal disease, and cognitive decline in the Atherosclerosis Risk in Communities (ARIC) study. Community Dent Oral Epidemiol. 2015 Feb;43(1):47-57.

14 Naorungroj S, Schoenbach VJ, Beck J, Mosley TH, Gottesman RF, Alonso A, et al. Crosssectional associations of oral health measures with cognitive function in late middle-aged adults: a community-based study. J Am Dent Assoc. 2013 Dec;144(12):1362-71.

15 Wu B, Plassman BL, Liang J, Wei L. Cognitive function and dental care utilization among community-dwelling older adults. Am J Public Health. 2007 Dec;97(12):2216-21.

16 Moriya S, Tei K, Murata A, Yamazaki Y, Hata $\mathrm{H}$, Muramatsu M, et al. Associations between self-assessed masticatory ability and higher brain function among the elderly. J Oral Rehabil. 2011 Oct;38(10):746-53.

17 Scherder E, Posthuma W, Bakker T, Vuijk PJ, Lobbezoo F. Functional status of masticatory system, executive function and episodic memory in older persons. J Oral Rehabil. 2008 May;35(5):324-36.

18 Saxe SR, Snowdon DA, Wekstein MW, Henry RG, Grant FT, Donegan SJ, et al. Dental amalgam and cognitive function in older women: findings from the Nun Study. J Am Dent Assoc. 1995 Nov;126(11):1495-501.
19 Takada K, Ura C, Takei N, Takeda K, Morishima S, Ishii T, et al. Effects of oral self-care on oral, cognitive, and daily performance functions in rural community-dwelling older people with mild cognitive impairment. Asian J Gerontol Geriatr. 2018;13(1):19-24.

20 Bergdahl M, Habib R, Bergdahl J, Nyberg L, Nilsson LG. Natural teeth and cognitive function in humans. Scand J Psychol. 2007 Dec; 48(6):557-65.

21 Starr JM, Hall RJ, Macintyre S, Deary IJ, Whalley LJ. Predictors and correlates of edentulism in the healthy old people in Edinburgh (HOPE) study. Gerodontology. 2008 Dec; 25(4):199-204.

22 Hansson P, Sunnegårdh-Grönberg K, Bergdahl J, Bergdahl M, Nyberg L, Nilsson LG. Relationship between natural teeth and memory in a healthy elderly population. Eur J Oral Sci. 2013 Aug;121(4):333-40.

23 Naorungroj S, Slade GD, Beck JD, Mosley TH, Gottesman RF, Alonso A, et al. Cognitive decline and oral health in middle-aged adults in the ARIC study. J Dent Res. 2013 Sep;92(9): 795-801.

24 Nilsson H, Berglund J, Renvert S. Tooth loss and cognitive functions among older adults. Acta Odontol Scand. 2014 Nov;72(8):639-44.

25 Steinmassl PA, Steinmassl O, Kraus G, Dumfahrt $\mathrm{H}$, Grunert I. Is cognitive status related to oral hygiene level and appropriate for determining need for oral hygiene assistance? J Periodontol. 2016 Jan;87(1):41-7.

26 Stewart R, Weyant RJ, Garcia ME, Harris T, Launer LJ, Satterfield S, et al. Adverse oral health and cognitive decline: the health, aging and body composition study. J Am Geriatr Soc. 2013 Feb;61(2):177-84.

27 Moriya S, Tei K, Toyoshita Y, Koshino H, Inoue N, Miura H. Relationship between periodontal status and intellectual function among community-dwelling elderly persons. Gerodontology. 2012 Jun;29(2):e368-74.

28 Kamer AR, Morse DE, Holm-Pedersen P, Mortensen EL, Avlund K. Periodontal inflammation in relation to cognitive function in an older adult Danish population. J Alzheimers Dis. 2012;28(3):613-24.

29 Wu B, Plassman BL, Crout RJ, Liang J. Cognitive function and oral health among community-dwelling older adults. J Gerontol A Biol Sci Med Sci. 2008 May;63(5):495-500.

$30 \mathrm{Yu} \mathrm{YH,} \mathrm{Kuo} \mathrm{HK}$. Association between cognitive function and periodontal disease in older adults. J Am Geriatr Soc. 2008 Sep;56(9): 1693-7.
31 Noble JM, Borrell LN, Papapanou PN, Elkind MS, Scarmeas N, Wright CB. Periodontitis is associated with cognitive impairment among older adults: analysis of NHANES-III. J Neurol Neurosurg Psychiatry. 2009 Nov;80(11): 1206-11.

32 Matthews JC, You Z, Wadley VG, Cushman M, Howard G. The association between selfreported tooth loss and cognitive function in the REasons for Geographic And Racial Differences in Stroke study: an assessment of potential pathways. J Am Dent Assoc. 2011 Apr; 142(4):379-90.

33 Stewart R, Sabbah W, Tsakos G, D'Aiuto F, Watt RG. Oral health and cognitive function in the Third National Health and Nutrition Examination Survey (NHANES III). Psychosom Med. 2008 Oct;70(8):936-41.

34 Stein PS, Kryscio RJ, Desrosiers M, Donegan SJ, Gibbs MB. Tooth loss, apolipoprotein E, and decline in delayed word recall. J Dent Res. 2010 May;89(5):473-7.

35 Tsakos G, Watt RG, Rouxel PL, de Oliveira C, Demakakos P. Tooth loss associated with physical and cognitive decline in older adults. J Am Geriatr Soc. 2015 Jan;63(1):91-9.

36 Reyes-Ortiz CA, Luque JS, Eriksson CK, Soto L. Self-reported tooth loss and cognitive function: data from the Hispanic established populations for epidemiologic studies of the elderly (Hispanic EPESE). Colomb Med (Cali). 2013 Sep;44(3):139-45.

37 Rozas NS, Sadowsky JM, Jeter CB. Strategies to improve dental health in elderly patients with cognitive impairment: A systematic review. J Am Dent Assoc. 2017 Apr;148(4):236245.e3.

38 Jaywant A, Toglia J, Gunning FM, O’Dell MW. The clinical utility of a 30-minute neuropsychological assessment battery in inpatient stroke rehabilitation. J Neurol Sci. 2018 Jul;390:54-62.

39 Roy S, Ficarro S, Duberstein P, Chapman BP, Dubovsky S, Paroski M, et al. Executive function and personality predict instrumental activities of daily living in Alzheimer disease. Am J Geriatr Psychiatry. 2016 Nov;24(11): 1074-83.

40 Henry JD, Phillips LH, Ruffman T, Bailey PE. A meta-analytic review of age differences in theory of mind. Psychol Aging. 2013 Sep; 28(3):826-39.

41 Henry JD, von Hippel W, Molenberghs P, Lee T, Sachdev PS. Clinical assessment of social cognitive function in neurological disorders. Nat Rev Neurol. 2016 Jan;12(1):28-39. 